\title{
Establishment of porcine and monkey colonic organoids for drug toxicity study
}

\author{
Haonan Li ${ }^{1}$, Yalong Wang ${ }^{1}$, Mengxian Zhang ${ }^{1}$, Hong Wang ${ }^{2}$, Along Cui ${ }^{3,4}$, Jianguo Zhao ${ }^{3,4,5}$, Weizhi Ji ${ }^{2}$ and \\ Ye-Guang Chen ${ }^{1,6^{*}}$ (1)
}

\begin{abstract}
Pig and monkey are widely used models for exploration of human diseases and evaluation of drug efficiency and toxicity, but high cost limits their uses. Organoids have been shown to be promising models for drug test as they reasonably preserve tissue structure and functions. However, colonic organoids of pig and monkey are not yet established. Here, we report a culture medium to support the growth of porcine and monkey colonic organoids. Wnt signaling and PGE2 are important for long-term expansion of the organoids, and their withdrawal results in lineage differentiation to mature cells. Furthermore, we observe that porcine colonic organoids are closer to human colonic organoids in terms of drug toxicity response. Successful establishment of porcine and monkey colonic organoids would facilitate the mechanistic investigation of the homeostatic regulation of the intestine of these animals and is useful for drug development and toxicity studies.
\end{abstract}

Keywords: Pig, Monkey, Colonic organoids, Culture, Drug toxicity

\section{Background}

Rodents, especially mice, have been widely used in biomedical research. However, mice do not exhibit the similar pathological features of human gastrointestinal disease. For instance, Adenomatous polyposis coli (APC) mutation-associated polyps are usually found in the colorectum in human, while APC-induced polyp formation occurs preferably in the small intestine in mice (Boivin et al. 2003; Flisikowska et al. 2012; Moser et al. 1990). Pig and cynomolgus monkey are regarded as ideal animal models to investigate the human gastrointestinal function and disease based on the similarity of genomic sequence, anatomic morphology and drug metabolism with human beings (Bray et al. 2018; Cibelli et al. 2013; Deglaire and Moughan 2012; Kararli 1995; Patterson et al. 2008; Ziegler et al. 2016). Multiple gastrointestinal disease models are established in pigs, such as short-bowel

\footnotetext{
${ }^{*}$ Correspondence: ygchen@tsinghua.edu.cn

${ }^{1}$ The State Key Laboratory of Membrane Biology, Tsinghua-Peking Center for Life Sciences, School of Life Sciences, Tsinghua University, Beijing 100084, China

Full list of author information is available at the end of the article
}

syndrome (Pereira-Fantini et al. 2011; Vegge et al. 2013) and colorectal cancer (Flisikowska et al. 2012). Accordingly, pigs have been approved for pharmaceutical testing by United States Food and Drug Administration (Gonzalez et al. 2015).

In the last decade, organoids have been demonstrated to be a great model for disease study, drug test and regeneration medicine. Organoids derived from adult stem cells possess the capability to maintain self-renewal while being able to differentiate into functional cell types, mimic the three-dimension (3D) structures and retain the functions of the origin tissues (Schutgens and Clevers 2020; Zhang et al. 2020). In 2009, Clevers and colleagues developed the first meaningful mouse intestinal organoids embedded in Matrigel with the culture medium supplemented with epidermal growth factor (EGF), Noggin and R-spondin (Sato et al. 2009). Subsequently, mouse colonic organoids and human intestinal organoids were also established successfully (Fujii et al. 2018; Jung et al. 2011; Sato et al. 2011). By far, canine, bovine, porcine, bat, feline, chicken intestinal organoids have been cultured successfully (Chandra et al. 2019; Derricott et al. 
2019; Gonzalez et al. 2013; Khalil et al. 2016; Kramer et al. 2020; Powell and Behnke 2017; Zhou et al. 2020). However, in our best knowledge, the porcine and monkey colonic organoid models are not reported. Here, we report the establishment of the organoids derived from the colon of pig and monkey. Using these organoids, we carried out drug toxicity studies and found that porcine and human colonic organoids exhibited similar toxicity response. These organoid models provide a useful platform to expand the species investigation and drug toxicity studies.

\section{Results}

Establishment of porcine and monkey colonic organoids

Fresh crypts from adult porcine colon were harvested and embedded into Matrigel and cultured with expansion medium (EM) based on previously reported human colonic organoid medium (Jung et al. 2011). The sphere organoids were observed without budding structures after 1 week (Fig. 1A), and these organoids were maintained for at least 16 passages about 3 months. The size of porcine colonic organoids (PCOs) was increased after several passages, indicating fast proliferation in

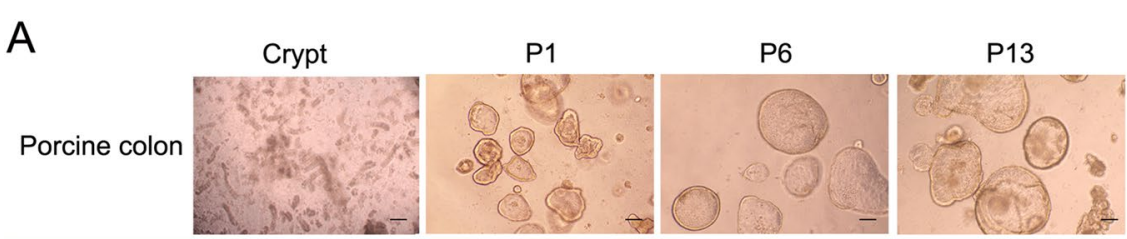

$\mathrm{B}$

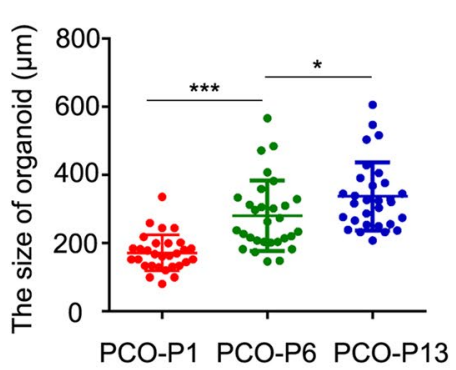

D

C

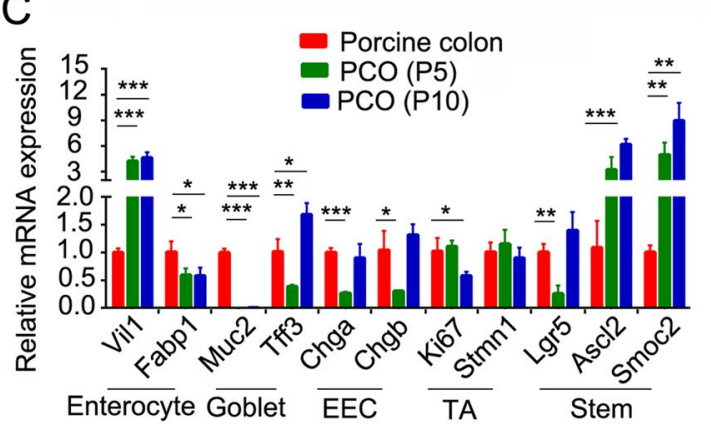

P1

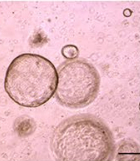
P13

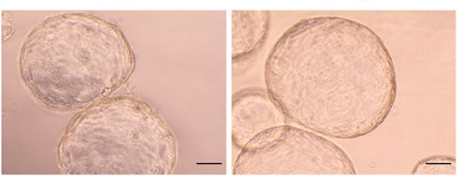

E

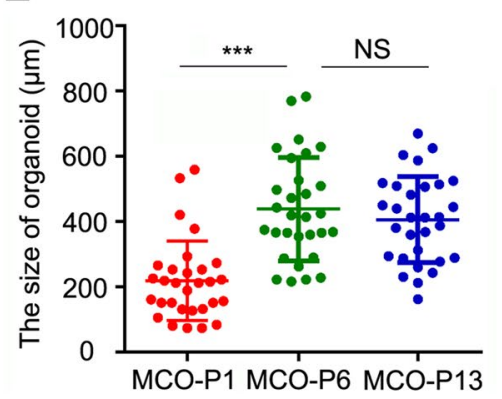

$\mathrm{F}$

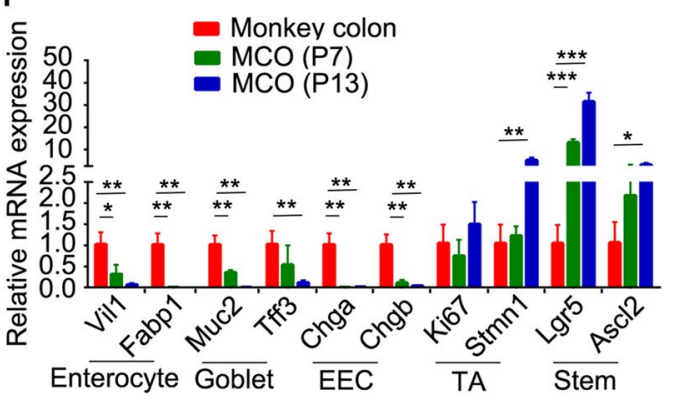

Fig. 1 Establishment of porcine and monkey colonic organoids. A, D Representative images of organoid growth of porcine colon (A) and monkey colon (D) in expansion medium from different passages. B, E Quantitation of the size of porcine colonic organoids (PCOs) (B) and monkey colonic organoids (MCOs) (E) in different passages. $\mathbf{C}, \mathbf{F}$ Expression of cell marker genes was examined with q-PCR in primary colon tissue and organoids from different passages in pig $(\mathbf{C})$ and monkey $(\mathbf{F})$. Scale bars, $100 \mu \mathrm{m} .{ }^{*} p<0.05,{ }^{* *} p<0.01,{ }^{* * *} p<0.001$. Data are displayed as the mean $\pm \mathrm{SD}$ by one-way ANOVA (B and $\mathbf{E}$ ) and Student's t-test $(\mathbf{C}$ and $\mathbf{F})$. 30 organoids were calculated for the size in $\mathbf{B}$ and $\mathbf{E}$. Three independent experiments were performed in $\mathbf{C}$ and $\mathbf{F}$ 
EM (Fig. 1B). The expression of trans-amplifying (TA) cell markers was similar between PCOs and the colon tissue. Meanwhile, the expression of stem cell marker genes (especially for $A s c l 2$ and $S m o c 2$ ) was significantly increased in PCOs compared to colon tissue, indicating the enrichment of stem cells in PCOs (Fig. 1C) (Munoz et al. 2012; van der Flier et al. 2009). The marker genes of mature cell types including enterocytes (marked by Fabp1), goblet cells (marked by Muc2 and Tff3), enteroendocrine cells (EECs) (marked by Chga and Chgb) had decreased expression in early passages of PCOs compared to the colon tissue, and their expression recovered (especially for EEC marker genes) in late passages of PCOs except Muc2 (Fig. 1C). Vil1 (villin1) mainly marks enterocytes, but is also expressed in other immature epithelial cells in the intestine (el Marjou et al. 2004). The expression of Vil1 was obviously increased in PCOs, confirming the epithelial identity of PCOs.

The similar culture procedure was conducted to culture organoids from adult cynomolgus monkey colon. EM also supported the growth of monkey colonic organoids (MCOs) (Fig. 1D), which could be maintained for at least 15 passages about 75 days. Fast proliferation was detected as the organoid size was significantly increased before passage 6 , and then the organoid size was stable (Fig. 1E). The expression of the maker genes for various cell types was compared between MCOs and primary tissues. The expression of enterocytes, goblet cells marker genes was significantly reduced in organoids compared to colon tissue, and EECs marker Chga and Chgb were rarely detected in the organoids (Fig. 1F). However, the stem cells and TA cells markers exhibited increased expression compared to primary monkey colon tissue. Together, EM could support porcine and monkey colonic organoid growth for long time and maintain the proliferation ability.

\section{Colonic organoids undergo differentiation in the differentiation medium}

Both PCOs and MCOs were in a high proliferation state with less differentiated mature cell types in EM. The homostatic balance of proliferating vs. differentiation cells in the intestine epithelium tissue is achieved with combinatory effect of various niche factors (Zhang et al. 2020; Zhu et al. 2021). To recapitulate the physiological cell constitution, we developed three differentiation media: (1) Differentiation-1 (D-1): withdrawal of Wnt3aconditional medium (Wnt3a-CM) and PGE2 from EM and the concentration of CHIR-99021 was decreased to $2.5 \mu \mathrm{M}$; (2) D-2: withdrawal of Wnt3a-CM, PGE2 and CHIR-99021; (3) D-3: withdrawal of Wnt3a-CM, PGE2, CHIR-99021, nicotinamide and SB202190. Then PCOs grown in EM were transferred into these differentiation media. Solid or budding organoids were found in D-1, -2 , -3 media at day 5 (Fig. 2A). However, organoids started to die in D-2 and -3 media in 1 to 2 weeks, while the organoids in D-1 could be maintained for at least 3 weeks. Since Wnt signaling plays a critical role in intestinal stem cell self-renewal (Barker 2014; Qi and Chen 2015; Sato and Clevers 2013; Yin et al. 2014), reducing Wnt signaling in differentiation media would lead to decreased organoid proliferation and cell death in long-term culture. Therefore, D-1 medium (DM) was used for the subsequent studies.

Compared to the PCOs growing in EM, morphological changes were observed in DM from sphere to budding organoids (Fig. 2A-B). The expression of mature cell types markers (including Vil1, Fabp1 for enterocytes; Muc2, Tff3 for goblet cells; Chga, Chgb for EECs) was significantly increased in DM compared to EM (Fig. 2C). However, Fabp 1 and $M u c 2$ expression remained low even in the PCOs cultured in DM compared to tissue, indicating an incomplete differentiation process in PCOs. The expression of TA cell markers (Ki67 and Stmn1) was not changed or slightly increased, and the stem cell markers (Lgr5 and Smoc2) in EM returned to the levels close to ones in the tissue. Reducing Wnt signaling in DM could directly counts for the decreased Lgr5 expression in PCOs. To further verify mature cell types in the DM-cultured PCOs, immunofluorescence staining for enterocytes, EECs and goblet cells were performed. As shown in Fig. 2D, Fabp1 ${ }^{+}$enterocytes were detected in the DM-cultured organoids, but were barely observed in the EM-culture organoids. Similarly, $\mathrm{ChgA}^{+}$EECs were significantly increased with DM culture (Fig. 2E). Furthermore, more $\mathrm{Muc}^{+}$goblet cells were observed in the DM-cultured organoids, as shown by immunofluorescence and Alcian blue staining (Fig. 2F-G). These data demonstrate that differentiation was promoted in DMcultured PCOs, which can better resemble the composition of the colonic epithelium.

To obtain more differentiated mature cells in MCOs, the organoids cultured in EM were transferred into DM for 1 week, and the size of sphere organoids was found to be smaller than the organoids in EM with the appearance of solid organoids (Fig. 3A-B). Accordingly, the expression of TA cells and stem cells markers was significantly decreased to the levels close to the tissue in DM-cultured MCOs, whereas some of differentiated cells markers Vil1 (enterocytes) and Tff3 (goblet cells) were upregulated compared to EM (Fig. 3C). Other differentiation markers were slightly increased in the DM-cultured MCOs compared to EM, but still lower than ones in the tissue, suggesting insufficient differentiation process in MCOs. Immunofluorescence staining 

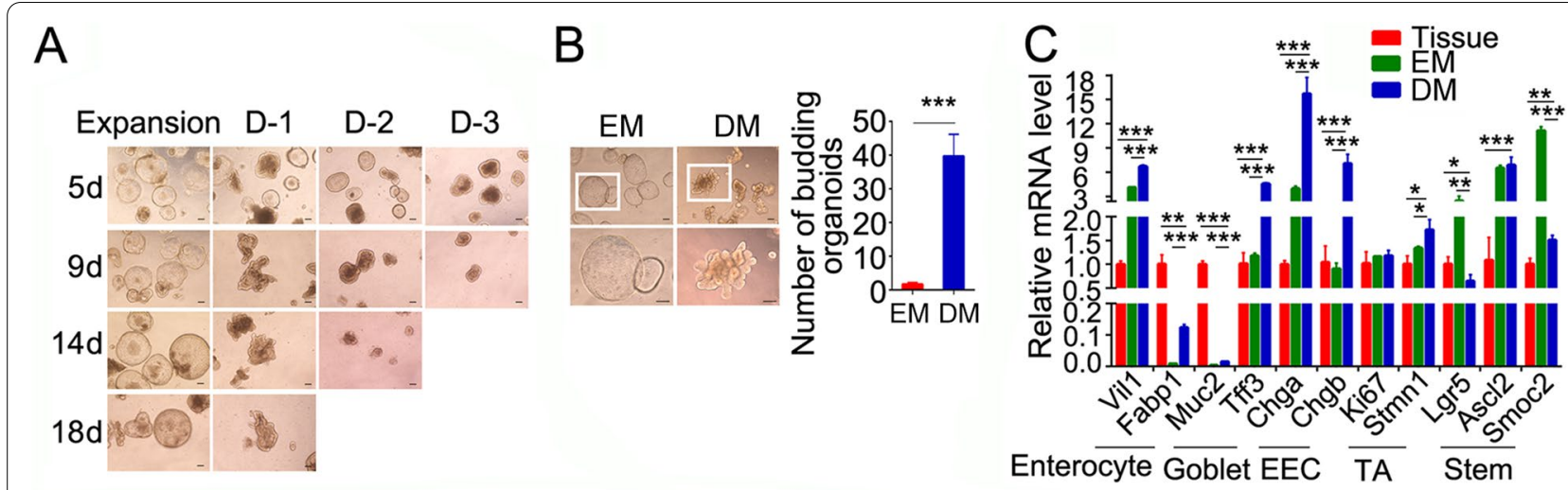

D
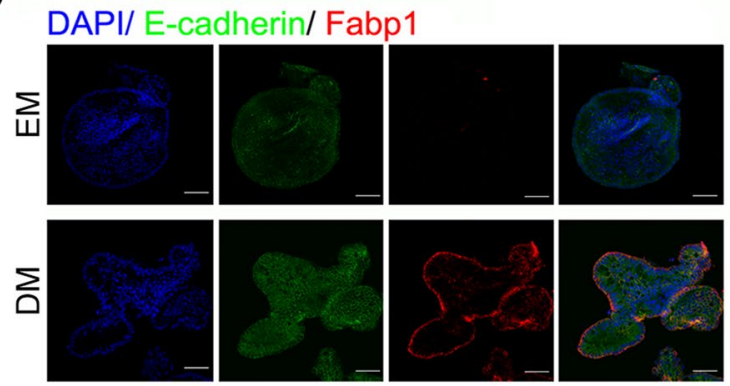

$\mathrm{F}$

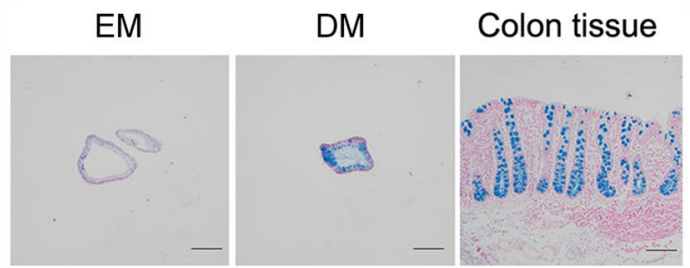

E

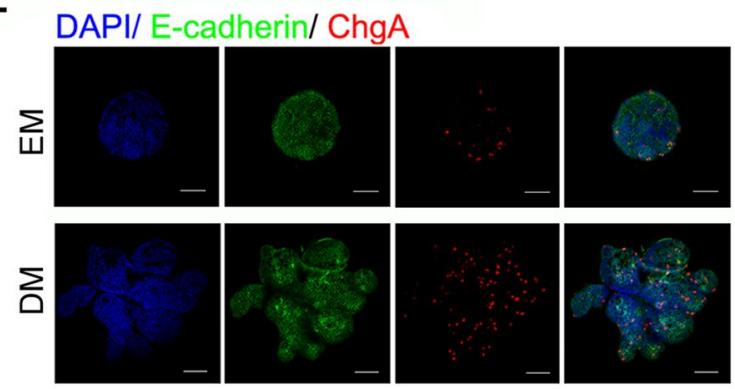

G DAPI/ E-cadherin/ Muc2
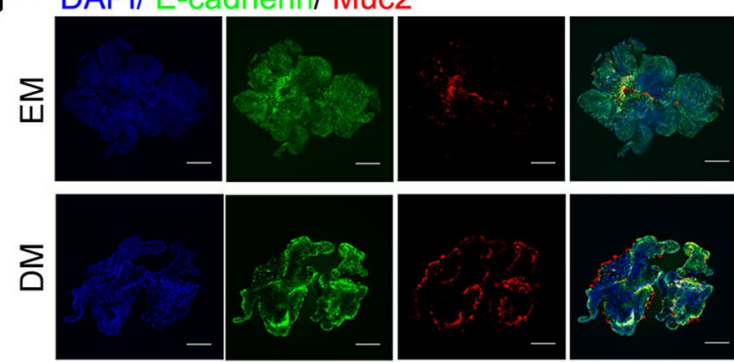

Fig. 2 Mature lineages of porcine colonic organoids are induced in differentiation medium. A Representative images of porcine colonic organoids grown in different differentiation media. Differentiation medium-1 (D-1): withdrawal of Wnt3a-CM and PGE2 from expansion medium with $2.5 \mu M$ CHIR-99021; Differentiation medium-2 (D-2): withdrawal of Wnt3a-CM, PGE2 and CHIR-99021 from expansion medium; Differentiation medium-3 (D-3): withdrawal of Wnt3a-CM, PGE2, CHIR-99021, nicotinamide and SB202190 from expansion medium. B Representative bright-field images and quantitation of the budding numbers of organoids in porcine colonic organoids cultured in expansion or differentiation medium for 7 days. White box depicts higher magnification below. C Expression of cell marker genes in proliferating (EM), differentiated (DM) porcine colonic organoids (passage 15) and tissue. D-G Fabp1 staining (D), ChgA staining (E), Alcian blue staining (F), Muc2 staining (G) in porcine colonic organoids cultured in expansion or differentiation medium for 7 days. Scale bars, $100 \mu \mathrm{m} .{ }^{*} p<0.05,{ }^{* *} p<0.01,{ }^{* * *} p<0.001$ analyzed by Student's t-test. Data are shown as mean $\pm \operatorname{SD}(n=3$ independent experiments)

further confirmed increased Fabp $1^{+}$enterocytes and Muc2 ${ }^{+}$goblet cells in the DM-cultured MCOs (Fig. 3DE). These results together indicate that this differentiation medium promotes the differentiation of enterocytes and goblet cells, but not very effective on EECs differentiation, which may suggest the species difference in cell differentiation regulation.

\section{Porcine colonic organoids are a promising model for drug} sensitivity test

Organoids are powerful tools to investigate the drug metabolism and toxicity (Burtin et al. 2020; Derricott et al. 2019). Most drug toxicity studies in organoids are carried out with the ones derived from mouse and human (Morizane et al. 2015; Park et al. 2019). Here, we applied porcine, monkey and human colonic 
A
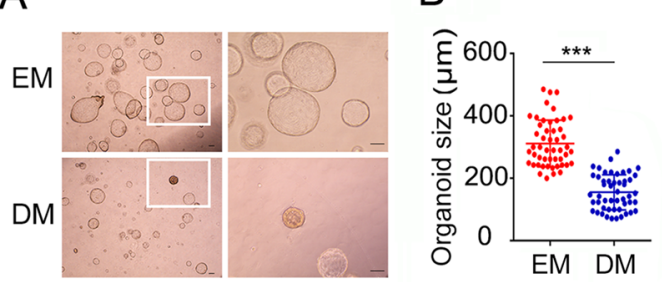

C

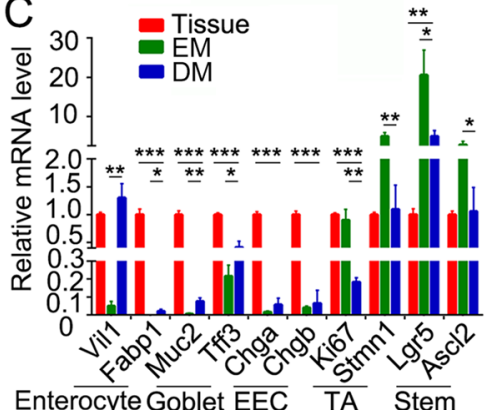

D

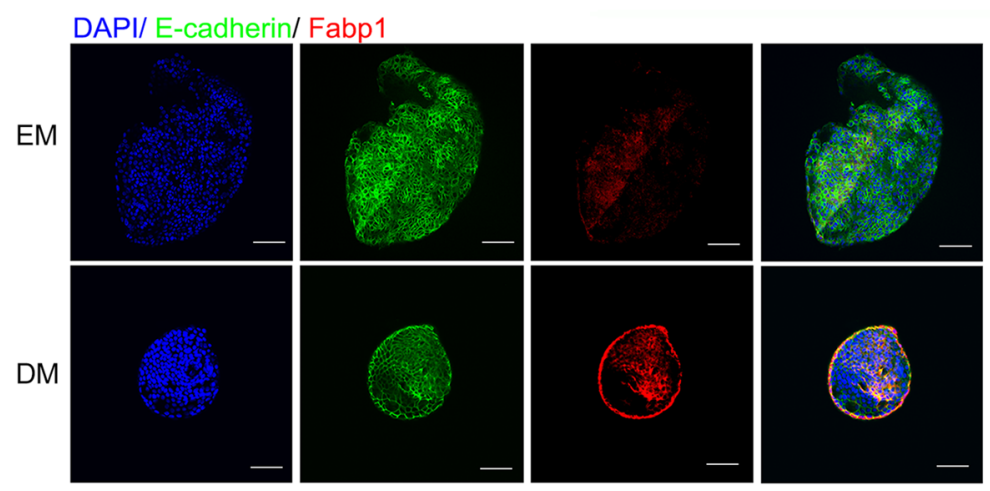

$\mathrm{E}$

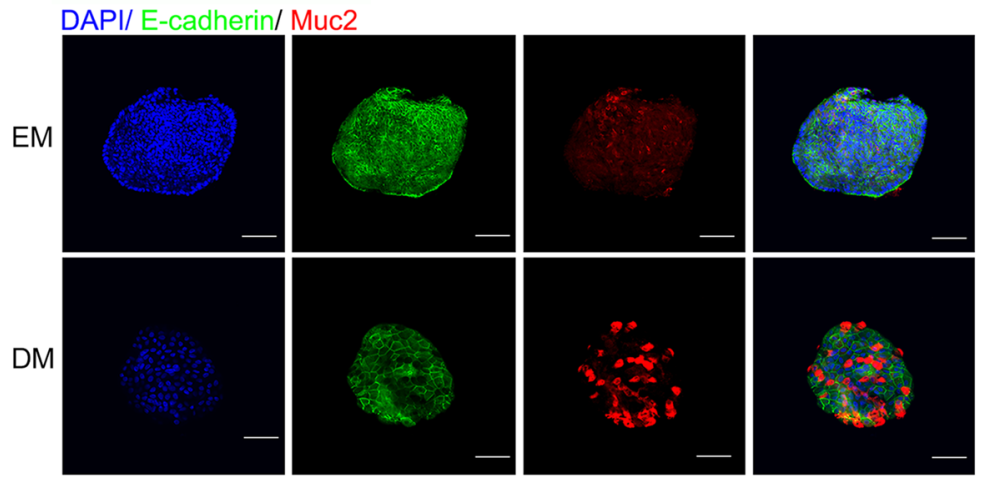

Fig. 3 Mature lineages of monkey colonic organoids are induced in differentiation medium. A, B Representative images (A) and quantitation of organoid size (B) of monkey colonic organoids cultivated in expansion or differentiation medium for 7 days. White box depicts higher magnification below. C Expression of cell marker genes in proliferating (EM), differentiated (DM) monkey colonic organoids (passage 15) and tissue. D, E Fabp1 staining (D), Muc2 staining (E) of monkey colonic organoids cultured with expansion or differentiation medium for 7 days. Scale bars, $100 \mu \mathrm{m}$. ${ }^{*} p<0.05,{ }^{* *} p<0.01,{ }^{* *} p<0.001$ analyzed by Student's t-test. Data are shown as mean $\pm \operatorname{SD}(n=3$ independent experiments)

organoids to test the toxicity response of anti-cancer drugs. Three types of organoids were seeded into 96 well plates in DM. Then, two clinically used anti-colon cancer drugs irinotecan (a DNA topoisomerase inhibitor) and regorafenib (a multi-targeted receptor tyrosine kinase inhibitor) were separately added into the organoids with various concentrations. After 4 days, the organoid growth and cell viability were examined. The organoid size and numbers were significantly reduced in porcine and human organoids treated with $1 \mu \mathrm{M}$ irinotecan. Inhibition on cell viability of human and porcine organoids was apparent at $5 \mu \mathrm{M}$, and there were rare viable organoids in porcine and human organoids treated with $50 \mu \mathrm{M}$ irinotecan (Fig. 4A). However, monkey organoids showed the resistance to irinotecan treatment: even in $50 \mu \mathrm{M}$ irinotecan, about $40 \%$ of the monkey organoids still survived. Regorafenib caused $80 \%$ cell death in porcine organoids in $1 \mu \mathrm{M}$, while monkey organoids were also more resistant to regorafenib (Fig. 4B). These data indicate that PCOs are more sensitive to these drugs than MCOs, and are closer to human colonic organoids. 

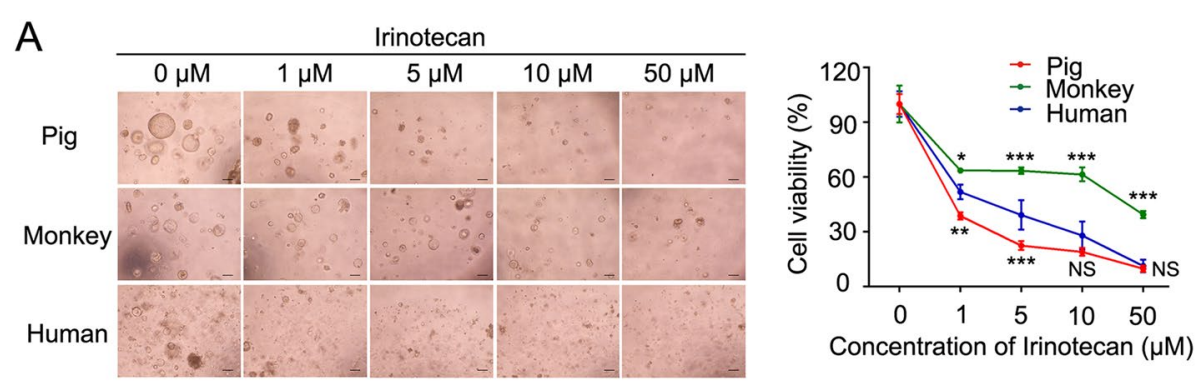

B
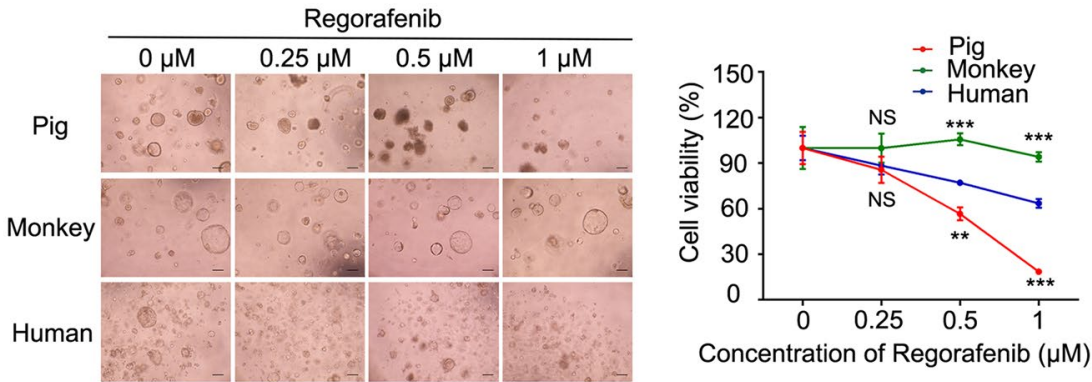

C
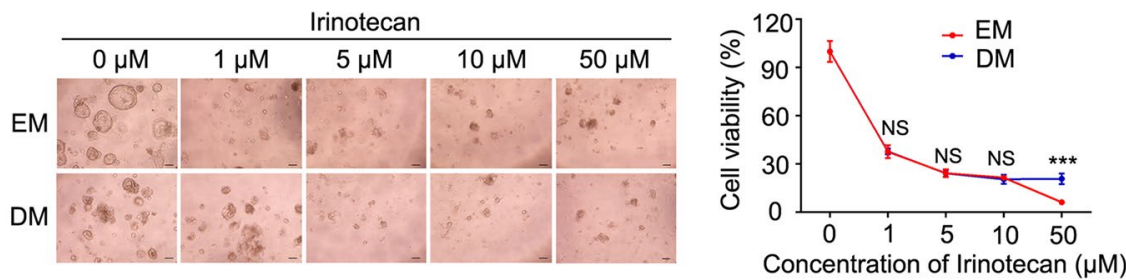

D
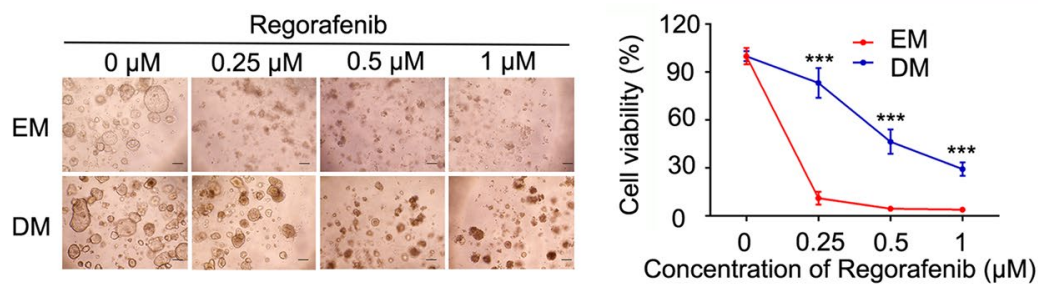

Fig. 4 Porcine colonic organoids are more sensitive to drug toxicity. A, B Representative bright-field images (left) and quantitation of cell viability (right) of porcine, monkey and human colonic organoids treated with different concentrations of irinotecan (A) or regorafenib (B) for 4 days. Data are shown as mean \pm SD ( $n=3$ independent experiments). C, D Representative bright-field images (left) and quantitation of cell viability (right) of PCOs in the expansion or differentiation medium treated with irinotecan $(\mathbf{C})$ or regorafenib (D) for 4 days. Data are shown as mean \pm SD by two-way ANOVA ( $n=3$ independent experiments). The significant difference was compared to human colonic organoids. ${ }^{*} p<0.05,{ }^{* *} p<0.01,{ }^{* * *} p<0.001$

To investigate the effect of culture conditions on drug sensitivity, we further compared the sensitivity of PCOs in EM or DM for 4 days to irinotecan or regorafenib. As shown in the following Fig. 4C and D, PCOs showed a similar toxicity response to irinotecan in two culture media. However, PCOs had different responses to regorafenib, and the differentiated PCOs were less sensitive, suggesting that highly proliferating cells are more sensitive to some drugs.

\section{Discussion}

In this study, we have successfully established porcine and monkey colonic organoid culture, and these organoids possessed the in vivo cell composition. Our results showed that reduced Wnt signaling and withdrawal of PGE2 induced lineage differentiation. However, when cultured in the differentiation medium for a long time, the organoids started to die. Therefore, there is a trade-off to achieve a balance between proliferation 
and differentiation in intestinal organoids. Withdrawal of Wnt signaling activity is necessary for enterocyte differentiation in human colonic organoids. The differentiation of goblet cells and EECs is promoted after withdrawal of nicotinamide and SB202190 (Sato et al. 2011). In addition, Notch inhibition could further promote goblet cell hyperplasia and cease proliferation (Otsuka et al. 2010; Sato et al. 2011). These reports provide clues to achieve balanced differentiation. In our differentiation system, D-1 could not only promote mature cell differentiation, but also maintain organoid growth for weeks. It provides a useful platform for functional studies, such as drug toxicity test. However, there is still a gap between differentiated organoids and tissue. D-2 and D-3 may have greater differentiated ability, while obvious growth inhibition and cell death limit their application. A more applicable differentiation condition needs to be explored.

Pig and monkey are among the animals frequently used for drug toxicity test (Dalgaard 2015). In our report, we found that PCOs may be closer to human colonic organoids in drug toxicity response. Our conclusion is in accordance with other studies to indicate that pigs are a good model for drug toxicity test. Firstly, the morphology of pig colon is very similar with human (Kararli 1995). The $\mathrm{pH}$ and transit time affecting drug bioavailability in pig colon are also comparable with that of human (Helke and Swindle 2013; Martinez et al. 2002). Secondly, minipigs have widely been accepted as models for toxicity testing of new medicines, especially in Europe (Bode et al. 2010; Ellegaard et al. 2010). Interestingly, the RETHINK project in the Europe has even decided to replace the dogs and non-human primates with minipigs for regulatory toxicity testing (Forster et al. 2010). Thirdly, the activity ratios of cytochrome P450, which mediates drug metabolism and is crucial for xenobiotics biotransformation and toxicity clearance, are closer in minipigs to human, compared to cynomolgus monkey (Dalgaard 2015; Turpeinen et al. 2007).

\section{Conclusions}

In summary, we demonstrated that the expansion medium could support the growth of both porcine and monkey colonic organoids for long-term cultivation. Reduced Wnt signaling and withdrawal of PGE2 induce mature cell differentiation. Furthermore, porcine and human colonic organoids are more similar in the drug sensitivity, suggesting that PCOs could be a better model for the evaluation of anti-colonic cancer drugs.

\section{Methods}

\section{Animals}

Bama miniature pigs used in this study were raised at the Beijing Farm Animal Research Center (affiliated to
Institute of Zoology, Chinese Academy of Sciences). The experiments involving pigs were approved by the Animal Ethics Committee of the Institute of Zoology, Chinese Academy of Sciences. The cynomolgus monkey (Macaca fascicularis) colon tissues were obtained from Yunnan Key Laboratory of Primate Biomedical Research, and experimental protocols were approved in advance by the Institutional Animal Care and Use Committee of Yunnan Key Laboratory of Primate Biomedical Research.

\section{Human colon tissue collection and ethics statement}

Human colon tissue was freshly obtained at least $10 \mathrm{~cm}$ away from the tumor border in surgically resected specimens at Peking University Third Hospital, Beijing, China, described before (Wang et al. 2020). All samples were obtained with informed consent, and this study was approved by the Peking University Third Hospital Medical Science Research Ethics Committee (M2018083), followed by relevant ethical regulations of Peking University Third Hospital Medical Science Research Ethics Committee.

\section{Isolation of crypts from porcine, monkey and human colon and organoid culture}

The colon tissue was extracted from three adult male pigs (6 months old) and two cynomolgus monkeys (14 years old) which were euthanized for research. The crypt isolation was conducted based on previous report (Zhao et al. 2015). Briefly, the colon tissue was cut longitudinally and washed by cold PBS for 3-4 times to remove the contaminant and feces. Then, adipose and vascular tissues were removed by operating scalpel. Small pieces of colon tissue (about $10 \mathrm{~cm}$ ) were incubated in $10 \mathrm{mM}$ EDTA in PBS for 30 min on ice. Next, the pieces were transferred into new PBS and the crypts were released by vigorously scrapping. The epithelial tissue was enriched by centrifugation ( $3 \mathrm{~min}$ at 1,000 rpm). The crypts were then embedded into Matrigel (BD Biosciences) and seeded on 24-well plate. After polymerization, the expansion medium was added. Advanced Dulbecco's Modified Eagle's Medium/F12 was supplemented with penicillin/ streptomycin, $2 \mathrm{mM}$ GlutaMAX, $1 \mathrm{mM} \mathrm{N}$-acetylcysteine, $1 \mathrm{X}$ N2, 1X B-27 to prepare a basal medium (all from Thermo Fisher). The expansion medium was supplemented with $50 \mathrm{ng} / \mathrm{mL}$ EGF (Invitrogen), $100 \mathrm{ng} / \mathrm{mL}$ Noggin (R\&D Systems), 500 ng/mL R-spondin-1 (R\&D Systems), $5 \mu \mathrm{M}$ CHIR-99021 (Selleck), $0.5 \mu \mathrm{M}$ A-8301 (Cayman), $10 \mu \mathrm{M}$ SB202190 (Selleck), $1 \mathrm{nM}$ Gastrin (Tocris), $10 \mu \mathrm{M}$ Y27632 (Enzo), $2.5 \mu \mathrm{M}$ PGE2 (Selleck), $10 \mathrm{mM}$ Nicotinamide (Sigma-Aldrich) and 30\%Wnt-conditional medium (Wnt3a-CM) (prepared from L-Wnt3a cell line (ATCC)) in basal medium. Growth medium was refreshed every 3-4 days. For passaging at 1:3-1:4 split 
ratios, the organoids were suspended with $1 \mathrm{ml}$ cold PBS after removal of medium and were pelleted by centrifugation (3 min at $300 \mathrm{~g}$ ). Then organoids were embedded into new Matrigel in defined medium as indicated in the figure legends. For differentiation experiment, the organoids in the expansion medium were split into new cell plates with the differentiation medium (omission of PGE2 and Wnt3a-CM from the expansion medium, 2.5 MM CHIR-99021) and analyzed after 7 days.

\section{Immunofluorescence}

Immunofluorescence was performed as previously described (Li et al. 2018). Briefly, organoids were fixed in $4 \%$ paraformaldehyde for $1 \mathrm{~h}$ at room temperature. Organoids were washed by PBS for 3 times and permeabilized by $0.5 \%$ Triton $\mathrm{X}-100$ for $1 \mathrm{~h}$ at room temperature. Then, samples were blocked with PBT solution (3\% BSA and $0.01 \%$ Triton X-100 in PBS) for $2 \mathrm{~h}$ at room temperature and incubated with primary antibodies overnight at $4{ }^{\circ} \mathrm{C}$. The fluorescein-labeled secondary antibodies (Life Technologies, 1:300) and 4', 6-diamidino-2-phenylindole (DAPI) were added for $1 \mathrm{~h}$ at room temperature next day. The following antibodies were used for immunofluorescence: rabbit anti-Fabp1 (Abcam, ab171739, 1:300); mouse anti-E-cadherin (B\&D, 610182, 1:300); rabbit antiMuc2 (Santa Cruze, sc-15334, 1:300); rabbit anti-ChgA (Abcam, ab15160, 1:300). The images were acquired from Olympus FV3000 Laser Scanning Microscope.

\section{Alcian blue staining}

The colon tissue was fixed with $4 \%$ formalin overnight and embedded in paraffin. The sections $(5 \mu \mathrm{m})$ were deparaffinized in isopropanol and graded alcohols. Then, sections were stained by Alcian blue staining kit (BASO) according to manufacturer's instructions. Sections were stained with Alcian blue for $15 \mathrm{~min}$ and nuclear fast red for $1 \mathrm{~min}$ (BA4087B, Baso). The images were obtained with a Nikon microscope.

\section{RNA extraction and quantitative RT-PCR}

The total RNA was extracted by RNeasy Mini Kit (Qiagen). The cDNA was obtained using Revertra Ace (Toyobo). Then, real-time PCR reactions were performed using qPCR Master Mix (Promega) in triplicates on a LightCycler 480 (Roche). The primers of selected gene were shown in Supplementary Table 1.

\section{Drug toxicity test}

Porcine, monkey and human colonic organoids were seeded into 96 well plates in the differentiation medium with different concentrations of drugs. The concentrations of irinotecan and regorafenib were set based on previous reports (Napolitano et al. 2015; Sim et al. 2018;
Yao et al. 2020). After 4 days, organoid growth was monitored with a microscope and cell viability was measured by Cell-Titer Glo 3D Cell Viability Assay (Promega, G9682) according to the manufacturer's instruction. CellTiter reagent $(25 \mu \mathrm{l})$ was added into each well and incubated with organoids for $30 \mathrm{~min}$ at room temperature. Then, the supernatant was collected after centrifugation (500 g, $1 \mathrm{~min}$ ) and luminescent signals were calculated by luminometer.

\section{Quantification and statistical analysis}

Student's t-test, one-way or two-way ANOVA analyses were used to compare difference between two groups as indicated in the figure legends. ${ }^{*} P<0.05,{ }^{* *} P<0.01$, ${ }^{* * *} P<0.001$. Data showed in column graphs indicated the mean \pm SD. The statistical analysis was carried in GraphPad Prism 6 software. The size of organoids was quantified with Image J. Each experiment was independently repeated at least three times.

\section{Supplementary Information}

The online version contains supplementary material available at https://doi. org/10.1186/s13619-021-00094-4.

Additional file 1.

Acknowledgements

We think all the lab members who have provided helps in this study.

Authors' contributions

$\mathrm{HL}$ and $\mathrm{YGC}$ conceived the experiments and wrote the manuscript. $\mathrm{HL}, \mathrm{YW}$ and $M Z$ performed the experiments. HW, AC, JZ and WJ helped with tissue collection. The author(s) read and approved the final manuscript.

Funding

This work was supported by grants from the National Key Research and Development Program of China (2017YFA0103601) and the National Natural Science Foundation of China (31730056 and 31988101) to YGC.

Availability of data and materials

All data generated or analyzed during this study are included in this published article and the supplementary material. Requests for materials should be addressed to the corresponding author.

\section{Declarations}

Ethics approval and consent to participate

The experiments involving Bama miniature pigs were approved by the Animal Ethics Committee of the Institute of Zoology, Chinese Academy of Sciences (IOZ20180061). The experimental protocols involving cynomolgus monkey (Macaca fascicularis) were approved in advance by the Institutional Animal Care and Use Committee of Yunnan Key Laboratory of Primate Biomedical Research (LPBR202004012). All human samples were obtained with informed consent, and this study was approved by the Peking University Third Hospital Medical Science Research Ethics Committee (M2018083), followed by relevant ethical regulations of Peking University Third Hospital Medical Science Research Ethics Committee.

Consent for publication

Not applicable. 


\section{Competing interests}

YGC is the Editor-in-Chief of Cell Regeneration. He was not involved in the review of decision related to this manuscript.

\section{Author details}

${ }^{1}$ The State Key Laboratory of Membrane Biology, Tsinghua-Peking Center for Life Sciences, School of Life Sciences, Tsinghua University, Beijing 100084, China. ${ }^{2}$ State Key Laboratory of Primate Biomedical Research, Institute of Primate Translational Medicine, Kunming University of Science and Technology, Kunming 650500, Yunnan, China. ${ }^{3}$ School of Life Sciences, University of Science and Technology of China, Hefei 230027, China. ${ }^{4}$ State Key Laboratory of Stem Cell and Reproductive Biology, Institute of Zoology, Chinese Academy of Sciences, Beijing 100101, China. ${ }^{5}$ University of Chinese Academy of Sciences, Beijing 100049, China. ${ }^{6}$ Max-Planck Center for Tissue Stem Cell Research and Regenerative Medicine, Guangzhou Regenerative Medicine and Health Guangdong Laboratory, Guangzhou 510700, China.

Received: 3 May 2021 Accepted: 2 September 2021 Published online: 02 October 2021

\section{References}

Barker N. Adult intestinal stem cells: critical drivers of epithelial homeostasis and regeneration. Nat Rev Mol Cell Biol. 2014;15:19-33.

Bode G, Clausing P, Gervais F, Loegsted J, Luft J, Nogues V, Sims J, Steering Group of the, R.P. The utility of the minipig as an animal model in regulatory toxicology. J Pharmacol Toxicol Methods. 2010;62:196-220.

Boivin GP, Washington K, Yang K, Ward JM, Pretlow TP, Russell R, Besselsen DG, Godfrey VL, Doetschman T, Dove WF, et al. Pathology of mouse models of intestinal cancer: consensus report and recommendations. Gastroenterology. 2003;124:762-77.

Bray F, Ferlay J, Soerjomataram I, Siegel RL, Torre LA, Jemal A. Global cancer statistics 2018: GLOBOCAN estimates of incidence and mortality worldwide for 36 cancers in 185 countries. CA Cancer J Clin. 2018;68:394-424.

Burtin F, Mullins CS, Linnebacher M. Mouse models of colorectal cancer: past, present and future perspectives. World J Gastroenterol. 2020;26:1394-426.

Chandra L, Borcherding DC, Kingsbury D, Atherly T, Ambrosini YM, BourgoisMochel A, Yuan W, Kimber M, Qi Y, Wang Q, et al. Derivation of adult canine intestinal organoids for translational research in gastroenterology. BMC Biol. 2019;17:33.

Cibelli J, Emborg ME, Prockop DJ, Roberts M, Schatten G, Rao M, Harding J, Mirochnitchenko O. Strategies for improving animal models for regenerative medicine. Cell Stem Cell. 2013;12:271-4.

Dalgaard L. Comparison of minipig, dog, monkey and human drug metabolism and disposition. J Pharmacol Toxicol Methods. 2015;74:80-92.

Deglaire A, Moughan PJ. Animal models for determining amino acid digestibility in humans - a review. Br J Nutr. 2012;108(Suppl 2):S273-281.

Derricott H, Luu L, Fong WY, Hartley CS, Johnston LJ, Armstrong SD, Randle N, Duckworth CA, Campbell BJ, Wastling JM, et al. Developing a 3D intestinal epithelium model for livestock species. Cell Tissue Res. 2019;375:409-24.

el Marjou F, Janssen KP, Chang BH, Li M, Hindie V, Chan L, Louvard D, Chambon $P$, Metzger D, Robine S. Tissue-specific and inducible Cre-mediated recombination in the gut epithelium. Genesis. 2004;39:186-93.

Ellegaard L, Cunningham A, Edwards S, Grand N, Nevalainen T, Prescott M, Schuurman T, Steering Group of the, R.P. Welfare of the minipig with special reference to use in regulatory toxicology studies. J Pharmacol Toxicol Methods. 2010;62:167-83.

Flisikowska T, Merkl C, Landmann M, Eser S, Rezaei N, Cui X, Kurome M, Zakhartchenko V, Kessler B, Wieland $\mathrm{H}$, et al. A porcine model of familial adenomatous polyposis. Gastroenterology. 2012;143:1173-1175.e1177.

Forster R, Bode G, Ellegaard L, van der Laan JW. The RETHINK project on minipigs in the toxicity testing of new medicines and chemicals: conclusions and recommendations. J Pharmacol Toxicol Methods. 2010;62:236-42.

Fujii M, Matano M, Toshimitsu K, Takano A, Mikami Y, Nishikori S, Sugimoto $\mathrm{S}$, Sato T. Human intestinal organoids maintain self-renewal capacity and cellular diversity in niche-inspired culture condition. Cell Stem Cell. 2018;23:787-793.e786.
Gonzalez LM, Williamson I, Piedrahita JA, Blikslager AT, Magness ST. Cell lineage identification and stem cell culture in a porcine model for the study of intestinal epithelial regeneration. PLoS One. 2013;8:e66465.

Gonzalez LM, Moeser AJ, Blikslager AT. Porcine models of digestive disease: the future of large animal translational research. Transl Res. 2015;166:12-27.

Helke KL, Swindle MM. Animal models of toxicology testing: the role of pigs. Expert Opin Drug Metab Toxicol. 2013;9:127-39.

Jung P, Sato T, Merlos-Suárez A, Barriga FM, Iglesias M, Rossell D, Auer H, Gallardo M, Blasco MA, Sancho E, et al. Isolation and in vitro expansion of human colonic stem cells. Nat Med. 2011;17:1225-7.

Kararli TT. Comparison of the gastrointestinal anatomy, physiology, and biochemistry of humans and commonly used laboratory animals. Biopharm Drug Dispos. 1995;16:351-80.

Khalil HA, Lei NY, Brinkley G, Scott A, Wang J, Kar UK, Jabaji ZB, Lewis M, Martin MG, Dunn JC, et al. A novel culture system for adult porcine intestinal crypts. Cell Tissue Res. 2016;365:123-34.

Kramer N, Pratscher B, Meneses AMC, TschulenkW, Walter I, Swoboda A, Kruitwagen HS, Schneeberger K, Penning LC, Spee B, et al. Generation of differentiating and long-living intestinal organoids reflecting the cellular diversity of canine intestine. Cells. 2020;9(4):822.

Li Y, Liu Y, Liu B, Wang J, Wei S, Qi Z, Wang S, Fu W, Chen YG. A growth factorfree culture system underscores the coordination between Wnt and BMP signaling in Lgr5(+) intestinal stem cell maintenance. Cell Discov. 2018:4:49.

Martinez M, Augsburger L, Johnston T, Jones WW. Applying the biopharmaceutics classification system to veterinary pharmaceutical products. Part I: biopharmaceutics and formulation considerations. Adv Drug Deliv Rev. 2002;54:805-24.

Morizane R, Lam AQ, Freedman BS, Kishi S, Valerius MT, Bonventre JV. Nephron organoids derived from human pluripotent stem cells model kidney development and injury. Nat Biotechnol. 2015:33:1193-200.

Moser AR, Pitot HC, Dove WF. A dominant mutation that predisposes to multiple intestinal neoplasia in the mouse. Science. 1990;247:322-4.

Munoz J, Stange DE, Schepers AG, van de Wetering M, Koo BK, Itzkovitz S, Volckmann R, Kung KS, Koster J, Radulescu S, et al. The Lgr5 intestinal stem cell signature: robust expression of proposed quiescent " +4 " cell markers. EMBO J. 2012;31:3079-91.

Napolitano S, Martini G, Rinaldi B, Martinelli E, Donniacuo M, Berrino L, Vitagliano D, Morgillo F, Barra G, De Palma R, et al. Primary and acquired resistance of colorectal cancer to anti-EGFR monoclonal antibody can be overcome by combined treatment of regorafenib with cetuximab. Clin Cancer Res. 2015;21:2975-83.

Otsuka M, Kang YJ, Ren J, Jiang H, Wang Y, Omata M, Han J. Distinct effects of p38alpha deletion in myeloid lineage and gut epithelia in mouse models of inflammatory bowel disease. Gastroenterology. 2010;138:1255-1265. e1251-1259.

Park E, Kim HK, Jee J, Hahn S, Jeong S, Yoo J. Development of organoid-based drug metabolism model. Toxicol Appl Pharmacol. 2019;385:114790.

Patterson JK, Lei XG, Miller DD. The pig as an experimental model for elucidating the mechanisms governing dietary influence on mineral absorption. Exp Biol Med (Maywood). 2008;233:651-64.

Pereira-Fantini PM, Thomas SL, Wilson G, Taylor RG, Sourial M, Bines JE. Shortand long-term effects of small bowel resection: a unique histological study in a piglet model of short bowel syndrome. Histochem Cell Biol. 2011;135:195-202.

Powell RH, Behnke MS. WRN conditioned media is sufficient for in vitro propagation of intestinal organoids from large farm and small companion animals. Biol Open. 2017;6:698-705.

Qi Z, Chen YG. Regulation of intestinal stem cell fate specification. Sci China Life Sci. 2015;58:570-8.

Sato T, Clevers H. Growing self-organizing mini-guts from a single intestinal stem cell: mechanism and applications. Science. 2013;340:1190-4.

Sato T, Vries RG, Snippert HJ, van de Wetering M, Barker N, Stange DE, van Es $J \mathrm{H}$, Abo A, Kujala P, Peters PJ, et al. Single Lgr5 stem cells build crypt-villus structures in vitro without a mesenchymal niche. Nature. 2009;459:262-5.

Sato T, Stange DE, Ferrante M, Vries RG, Van Es JH, Van den Brink S, Van Houdt WJ, Pronk A, Van Gorp J, Siersema PD, et al. Long-term expansion of epithelial organoids from human colon, adenoma, adenocarcinoma, and Barrett's epithelium. Gastroenterology. 2011;141:1762-72.

Schutgens F, Clevers H. Human organoids: tools for understanding biology and treating diseases. Annu Rev Pathol. 2020;15:211-34. 
Sim JJ, Park MH, Baek JH, Lee H, Jeong KY, Kim HM. Investigation into Enhancing capecitabine efficacy in colorectal cancer by inhibiting focal adhesion kinase signaling. Anticancer Res. 2018;38:4667-76.

Turpeinen M, Ghiciuc C, Opritoui M, Tursas L, Pelkonen O, Pasanen M. Predictive value of animal models for human cytochrome P450 (CYP)-mediated metabolism: a comparative study in vitro. Xenobiotica. 2007;37:1367-77.

van der Flier LG, van Gijn ME, Hatzis P, Kujala P, Haegebarth A, Stange DE, Begthel H, van den Born M, Guryev V, Oving I, et al. Transcription factor achaete scute-like 2 controls intestinal stem cell fate. Cell. 2009;136:903-12.

Vegge A, Thymann T, Lund P, Stoll B, Bering SB, Hartmann B, Jelsing J, Qvist N, Burrin DG, Jeppesen PB, et al. Glucagon-like peptide-2 induces rapid digestive adaptation following intestinal resection in preterm neonates. Am J Physiol Gastrointest Liver Physiol. 2013;305:G277-285.

Wang Y, Song W, Wang J, Wang T, Xiong X, Qi Z, Fu W, Yang X, Chen YG. Singlecell transcriptome analysis reveals differential nutrient absorption functions in human intestine. J Exp Med. 2020;217(2):e20191130.

Yao Y, Xu X, Yang L, Zhu J, Wan J, Shen L, Xia F, Fu G, Deng Y, Pan M, et al. Patient-derived organoids predict chemoradiation responses of locally advanced rectal cancer. Cell Stem Cell. 2020:26:17-26.e16.
Yin X, Farin HF, van Es JH, Clevers H, Langer R, Karp JM. Niche-independent high-purity cultures of Lgr5+ intestinal stem cells and their progeny. Nat Methods. 2014;11:106-12.

Zhang M, Liu Y, Chen YG. Generation of 3D human gastrointestinal organoids: principle and applications. Cell Regen. 2020;9:6.

Zhao B, Qi Z, Li Y, Wang C, Fu W, Chen YG. The non-muscle-myosin-II heavy chain Myh9 mediates colitis-induced epithelium injury by restricting Lgr5+ stem cells. Nat Commun. 2015;6:7166.

Zhou J, Li C, Liu X, Chiu MC, Zhao X, Wang D, Wei Y, Lee A, Zhang AJ, Chu H, et al. Infection of bat and human intestinal organoids by SARS-CoV-2. Nat Med. 2020;26:1077-83.

Zhu G, Hu J, Xi R. The cellular niche for intestinal stem cells: a team effort. Cell Regen. 2021;10:1.

Ziegler A, Gonzalez L, Blikslager A. Large animal models: the key to translational discovery in digestive disease research. Cell Mol Gastroenterol Hepatol. 2016;2:716-24.

\section{Submit your manuscript to a SpringerOpen ${ }^{\circ}$ journal and benefit from:}

- Convenient online submission

- Rigorous peer review

- Open access: articles freely available online

- High visibility within the field

Retaining the copyright to your article

Submit your next manuscript at $\boldsymbol{\nabla}$ springeropen.com 